\title{
Combination of low-dose testosterone and vildagliptin confers cardioprotection in castrated obese rats
}

\author{
Apiwan Arinno1,2,3,*, Nattayaporn Apaijai1,2,*, Puntarik Kaewthep1,2,3, Wasana Pratchayasakul1,2,3, \\ Thidarat Jaiwongkam1,2, Sasiwan Kerdphoo1,2, Siriporn C Chattipakorn1,2,4 and Nipon Chattipakorn1,2,3
}

${ }^{1}$ Cardiac Electrophysiology Research and Training Center, Faculty of Medicine, Chiang Mai University, Chiang Mai, Thailand ${ }^{2}$ Center of Excellence in Cardiac Electrophysiology Research, Chiang Mai University, Chiang Mai, Thailand

${ }^{3}$ Cardiac Electrophysiology Unit, Department of Physiology, Faculty of Medicine, Chiang Mai University, Chiang Mai, Thailand 4Department of Oral Biology and Diagnostic Sciences, Faculty of Dentistry, Chiang Mai University, Chiang Mai, Thailand

Correspondence should be addressed to N Chattipakorn: nchattip@gmail.com

*(A Arinno and N Apaijai contributed equally to this work)

\begin{abstract}
Although a physiological dose of testosterone replacement therapy (p-TRT) has been shown to improve left ventricular (LV) function, some studies reported that it increased the risk of myocardial infarction in testosterone-deprived men. We previously reported that vildagliptin might be used as an alternative to p-TRT. In this study, we hypothesized that a combined low-dose TRT with vildagliptin exerts greater efficacy than single regimen in improving cardiometabolic function in obese, insulin-resistant rats with testosterone deprivation. Male rats were fed on a normal diet or high-fat diet for 12 weeks. Then, they were divided into two subgroups, sham operation and orchiectomy (normal diet rats with orchiectomy (NDO), high-fat diet rats with orchiectomy (HFO)) and fed their diets for another 12 weeks. At week 25, orchiectomized rats were subdivided into four groups: vehicle, p-TRT, vildagliptin and combined drugs. At week 29, cardiometabolic and biochemical parameters were determined. HFO rats had obese insulin resistance with a worse LV dysfunction, compared with sham. Vildagliptin and combined drugs effectively reduced insulin resistance. All treatments reduced blood pressure, cardiac autonomic imbalance, LV dysfunction, mitochondrial dysfunction, apoptosis and increased mitochondrial fusion in NDO and HFO rats. However, p-TRT and combined drugs, but not vildagliptin, reduced mitochondrial fission in NDO and HFO rats. We concluded that combined low-dose TRT with vildagliptin mitigated LV function at a similar level to the $\mathrm{p}$-TRT alone and vildagliptin via improving mitochondrial fusion, reducing mitochondrial dysfunction and apoptosis in testosterone-deprived rats. Our findings suggest that low-dose TRT combined with vildagliptin may be an alternative for p-TRT in conditions of obese insulin resistance with testosterone deprivation.
\end{abstract}
Key Words
- obesity
- testosterone deprivation
- left ventricular function
- mitochondria
- apoptosis

Journal of Endocrinology (2019) 240, 467-481 (c) 2019 Society for Endocrinology Published by Bioscientifica Ltd. Printed in Great Britain 


\section{Introduction}

The global prevalence of obesity is gradually increasing (Reilly et al. 2018), and long-term high-fat diet (HFD) consumption is one of several causes of obesity (Apaijai et al. 2012, Hruby \& Hu 2015). Our previous studies showed that 12 weeks of HFD consumption led to obesity, insulin resistance and dyslipidemia in rats (Pratchayasakul et al. 2011, Apaijai et al. 2012) and is associated with an increase in blood pressure, cardiac sympathovagal imbalance and LV dysfunction (Apaijai et al. 2012, Apaiajai et al. 2018). Moreover, we have shown that testosterone deprivation increased the severity of LV dysfunction in obese, insulinresistant rats (Apaiajai et al. 2018), and it is also associated with a worsening of cardiac mitochondrial dysfunction and cardiac apoptosis (Apaiajai et al. 2018).

A physiological dose of testosterone replacement therapy (TRT) has been shown to be beneficial in male patients who had hypogonadism (Byrne \& Nieschlag 2003, Bhasin etal.2018). In animal studies, a physiological dose of TRT improved cardiac contractility in orchiectomized rats (Eleawa et al. 2013), reduced LV dysfunction and infarct size in orchiectomized rats with cardiac ischemia/reperfusion injury (Pongkan et al. 2015) and promoted angiogenesis in orchiectomized rats with acute myocardial infarction (Chen et al. 2012). Results from a clinical study showed that a physiological dose of TRT increased mortality rate and risk of myocardial infarction in testosterone-deprived subjects with cardiovascular diseases (Finkle et al. 2014). Recently, several pharmacological interventions have been investigated as an alternative to testosterone (Pongkan et al. 2016, Bae et al. 2017). A dipeptidyl peptidase-4 (DPP4) inhibitor, vildagliptin, has been shown to improve both peripheral insulin resistance and LV function in a similar manner to the physiological dose of TRT without any changes in plasma testosterone levels in orchiectomized rats with obese insulin resistance (Pongkan et al. 2016). On the other hand, vildagliptin failed to prevent the cognitive impairment in those rats (Pintana et al. 2015). Thus, a combined low-dose TRT with vildagliptin may provide a great benefit in the treatment of obese subjects with testosterone deprivation. However, the effects of a combined low-dose TRT with vildagliptin on metabolic parameters and LV function have never been investigated. In this study, we hypothesized that the combined lowdose TRT with vildagliptin exerts greater efficacy than either single regimen in improving metabolic parameters and LV function via attenuating cardiac mitochondrial dysfunction and apoptosis in obese, insulin-resistant rats with testosterone deprivation.

\section{Materials and methods}

All experimental protocols in this study were approved by the Faculty of Medicine, Chiang Mai University Institutional Animal Care and Use committee (permit no. 17/2560); experimental procedures were carried out in compliance with NIH guidelines, and in accordance with the ARRIVE guidelines for reporting animal research.

\section{Animals and experimental design}

Male Wistar rats weighing between 180 and $200 \mathrm{~g}$ were obtained from Nomura Siam International company, Bangkok, Thailand $(n=60)$. Rats were housed in a room with controlled temperature $\left(25^{\circ} \mathrm{C}\right)$ and humidity, and they were allowed to acclimatize for 7 days prior to the experiment. Rats were randomly divided into two dietary groups ( $n=30$ /group) - a normal diet group (ND: a standard laboratory rat diet containing $19.77 \% \mathrm{E}$ fat) and a highfat diet group (HFD: a diet containing 59.28\% E fat). All rats could access both diet and water ad libitum. Rats were fed with their assigned diet for 12 weeks to induce obese insulin resistance in the HFD group (Pratchayasakul et al. 2011, Apaiajai et al. 2018). At week 13, rats in each dietary group were randomly divided into two operation groups: (1) sham operation (ND rats with sham operation; NDS and HFD rats with sham operation; HFS; $n=6 /$ group) and (2) orchiectomy (ORX; ND rats with orchiectomy (NDO) and HFD rats with orchiectomy (HFO); $n=24$ /group). A bilateral orchiectomy (ORX) was performed using a sterile technique as previously described (Pongkan et al. 2015, Apaiajai et al. 2018). After confirming testosterone deprivation aggravated LV dysfunction as previously described (Apaiajai et al. 2018), rats after 12 weeks of orchiectomy (ORX) were pharmacologically intervened (Apaiajai et al. 2018). Pharmacological interventions were given to the rats after 12 weeks of ORX. Thus, in week 25, NDO and HFO rats were subdivided into four pharmacological intervention groups as follows: (1) vehicle (NDOV and HFOV; rats were given castor oil via s.c. injection; $n=6 /$ group); (2) physiological dose of TRT (NDOT and HFOT; rats were given $2 \mathrm{mg} / \mathrm{kg}$ of testosterone via s.c. injection; $n=6$ /group) (Pintana et al. 2015, Pongkan et al. 2016); (3) DPP-4 inhibitor vildagliptin (NDOVil and HFOVil; rats were given $3 \mathrm{mg} / \mathrm{kg}$ of vildagliptin via gavage feeding) (Pintana et al. 2015, Pongkan et al. 2016) and (4) combined low-dose TRT and DPP-4 inhibitor (NDOVilT and HFOVilT; rats were given $1 \mathrm{mg} / \mathrm{kg}$ of testosterone via s.c. injection and $3 \mathrm{mg} / \mathrm{kg}$ of vildagliptin via gavage https://joe.bioscientifica.com

https://doi.org/10.1530/JOE-18-0673 (c) 2019 Society for Endocrinology Published by Bioscientifica Ltd. Printed in Great Britain 
feeding). NDS and HFS rats were given castor oil via s.c. injection. Rats were given their assigned interventions for 4 weeks, and then metabolic parameters and LV function were determined. After the invasive LV function was carried out at the end of the protocol, rats were decapitated. The heart was rapidly removed to determine the cardiac mitochondrial function and dynamics and the level of apoptosis. The experimental protocol is shown in Fig. 1.

\section{Determination of metabolic parameters}

Body weight and food intake were recorded weekly, and visceral fat weight was recorded after decapitation. The blood was collected from the tail tip, and centrifuged at $1077 \mathrm{~g}$ for $10 \mathrm{~min}$. The plasma was collected to allow the determination of plasma testosterone, insulin, glucose, triglyceride, total cholesterol and high-density lipoprotein cholesterol (HDL-C) levels (Apaiajai et al. 2018).

Plasma testosterone levels were determined using the ELISA technique at the Central Laboratory Service of Maharaj Nakorn Chiang Mai Hospital, Faculty of Medicine, Chiang Mai University. Plasma insulin levels were determined using a commercial ELISA kit (Merck Millipore). Plasma glucose, total cholesterol and triglyceride levels were determined using commercial colorimetric kits (Erba Mannheim, Mannheim, Germany). The degree of insulin resistance was assessed by the HOMA index, which was calculated from fasting plasma insulin and fasting plasma glucose concentration. Plasma HDL-C levels were determined using a commercial colorimetric kit (BioVision Inc., California, USA). Plasma low-density lipoprotein cholesterol (LDL-C) levels were calculated using the Friedewald formula (Apaiajai et al. 2018).

\section{Blood pressure determination and heart rate variability determination}

Systolic blood pressure (SBP) and diastolic blood pressure (DBP) were determined in conscious rats using a noninvasive volume-pressure recording tail cuff method (CODA-2, Kent scientific, Connecticut, USA) (Apaiajai et al. 2018).

Heart rate variability (HRV) was determined as described previously (Apaijai et al. 2013, Apaiajai et al. 2018). In brief, a lead II electrocardiogram (ECG) was

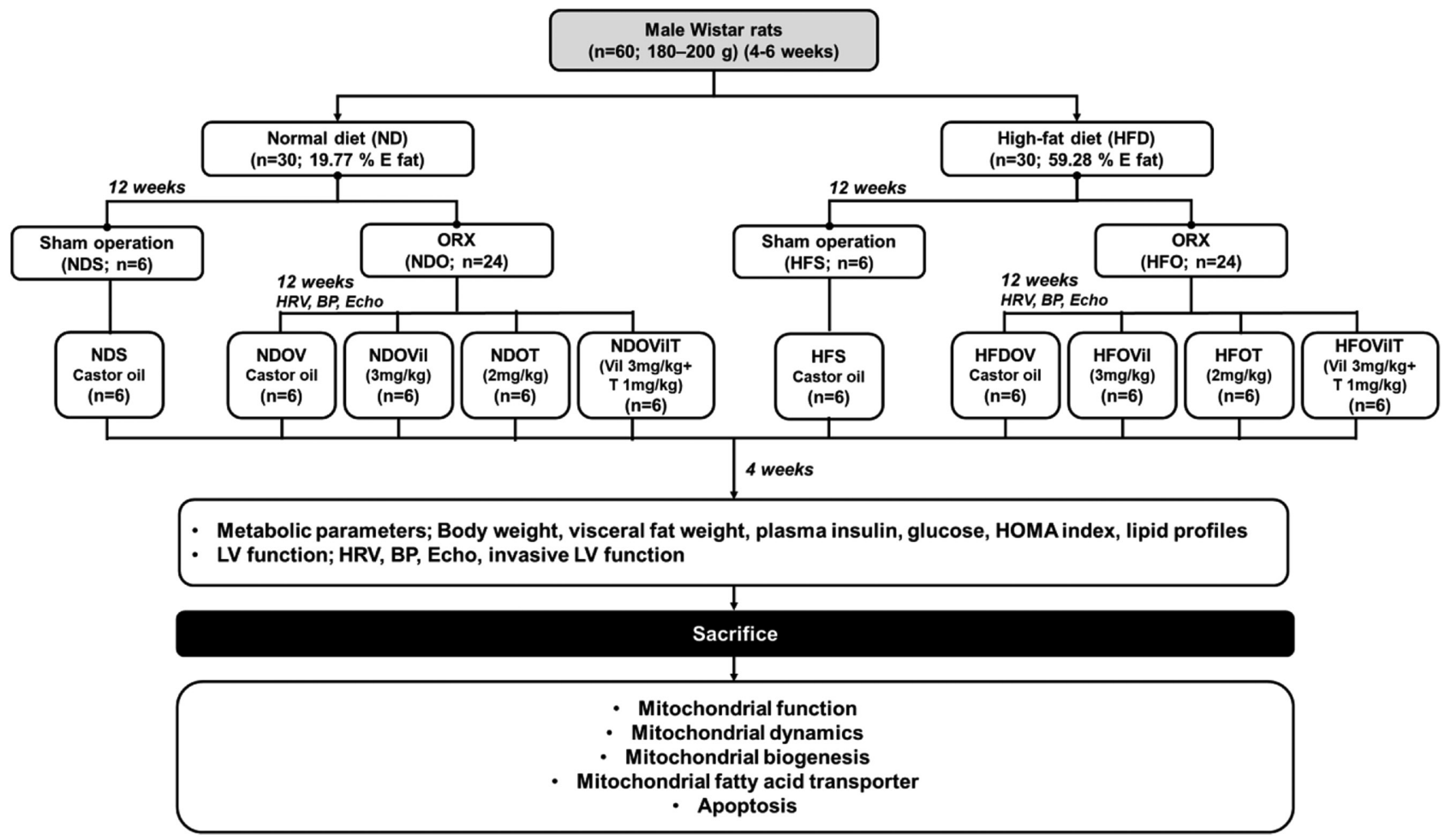

Figure 1

Experimental protocol. BP, blood pressure; DPP-4, dipeptidyl peptidase 4; HFD, high-fat diet; HFO, high-fat diet rats with testosterone deprivation; HRV, heart rate variability; LV, left-ventricle; ND, normal diet; NDO, normal diet rats with testosterone deprivation; ORX, orchiectomy; T, physiological dose testosterone replacement therapy; $\mathrm{V}$, vehicle; VilT, combined low-dose testosterone replacement therapy with vildagliptin. 
recorded in conscious rats for $20 \mathrm{~min}$ (PowerLab 4/25T, $\mathrm{AD}$ instruments, Sydney, Australia). The stable ECG was used to analyze the data. At least 300 consecutive RR intervals were chosen, and the power spectra of RR intervals were obtained using the Fast Fourier Transform algorithm. A high-frequency band (HF; $0.04-0.15 \mathrm{~Hz}$ ), a low-frequency band (LF; $0.15-0.4 \mathrm{~Hz}$ ) and a very-low frequency band (VLF $<0.15 \mathrm{~Hz}$ ) were detected. Then, the HF and LF were divided by the total power minus VLF in order to minimize the effect of changes in total power on both frequency bands. LF indicates a combination of sympathetic and parasympathetic activities, while $\mathrm{HF}$ indicates parasympathetic activity. The LF/HF ratio was used as an indicator for cardiac sympathovagal balance (Apaijai et al. 2013, Apaiajai et al. 2018).

\section{Determination of LV function}

Non-invasive LV function was determined using an echocardiograph. Rats were lightly anesthetized using $1 \%$ isoflurane. An S12 probe was placed on the chest at the parasternal short axis and connected to the machine (GE vivid-i, GE healthcare). The M-mode echocardiogram was recorded at the papillary levels. The \%LV ejection fraction (EF) was determined as an indicator of LV function (Apaiajai et al. 2018).

At the end of the treatment period, invasive LV function was determined using pressure-volume (P-V) loop analysis. Rats were deeply anesthetized using a combination of $50 \mathrm{mg} / \mathrm{kg}$-Zoletil (Virbac, Bangkok, Thailand) and $3 \mathrm{mg} / \mathrm{kg}$-Xylazine (LBS Labs, Bangkok, Thailand) via intramuscular injection. The right carotid artery was identified, and a P-V admittance catheter (1.9F VSL catheter; Transonic Scisense, NY, USA) was inserted through the right carotid artery and advanced into the $\mathrm{LV}$. Then, the P-V catheter was connected to the P-V recording system (ADV500, Transonic), and the signals were recorded via a Labscribe 2 program (iworx, $\mathrm{NH}, \mathrm{USA}$ ). LV function, including heart rate (HR), end-systolic and diastolic pressure (ESP, EDP), $\pm \mathrm{dP} / \mathrm{dt}$ and stroke volume (SV), was recorded and analyzed (Apaiajai et al. 2018).

\section{Cardiac mitochondrial function}

The heart was removed and used to determine the cardiac mitochondrial function. The heart tissue was minced and homogenized in an ice-cold buffer. The homogenates were subjected to differential centrifugation, and the cardiac mitochondria were obtained. The cardiac mitochondrial protein content was determined using a bicinchoninic acid (BCA) assay (Apaijai et al. 2013).

\section{Cardiac mitochondrial reactive oxygen species (ROS) determination}

Cardiac mitochondria $(0.4 \mathrm{mg} / \mathrm{mL})$ were stained with $2 \mu \mathrm{M}$ dichloro-dihydro-fluorescein diacetate (DCFH-DA) dye and incubated at $25^{\circ} \mathrm{C}$ for $20 \mathrm{~min}$. The fluorescence intensity of DCF was detected at $\lambda_{\text {ex }} 485 \mathrm{~nm}$ and $\lambda_{\text {em }}$ $530 \mathrm{~nm}$ using a fluorescent microplate reader (BioTek). An increased DCF fluorescence intensity indicates an increased cardiac mitochondrial ROS level (Apaijai et al. 2013).

\section{Cardiac mitochondrial membrane potential changes}

Cardiac mitochondria $(0.4 \mathrm{mg} / \mathrm{mL})$ were stained with $5 \mu \mathrm{M} \mathrm{JC}-1$ dye and incubated at $37^{\circ} \mathrm{C}$ for $30 \mathrm{~min}$. The JC-1 aggregation form was detected at $\lambda_{\mathrm{ex}} 485 \mathrm{~nm}$ and $\lambda_{\mathrm{em}}$ $590 \mathrm{~nm}$, and the JC- 1 monomer form was detected at $\lambda_{\mathrm{ex}}$ $485 \mathrm{~nm}$ and $\lambda_{\mathrm{em}} 530 \mathrm{~nm}$. The ratio of JC- 1 aggregation/JC- 1 monomer, that is, a red/green fluorescent intensity ratio, was used to indicate mitochondrial membrane potential changes. A decrease in a red/green fluorescence intensity ratio indicates mitochondrial membrane depolarization (Apaijai et al. 2013).

\section{Cardiac mitochondrial swelling}

Cardiac mitochondria $(0.4 \mathrm{mg} / \mathrm{mL})$ in a respiration buffer were used to determine cardiac mitochondrial swelling. The absorbance was detected at $540 \mathrm{~nm}$, and a reduction of the absorbance indicates cardiac mitochondrial swelling (Apaijai et al. 2013). Cardiac mitochondrial morphology was also investigated using a transmission electron microscope (TEM) (Apaijai et al. 2013).

\section{Cardiac mitochondrial respiration}

Cardiac mitochondrial respiration was assessed using a high-throughput automated 96-well extracellular flux analyzer (XFe96, Agilent seahorse, CA, USA). $0.5 \mathrm{mg} / \mathrm{mL}$ of cardiac mitochondrial protein was used, and mitochondria were suspended in a medium buffer containing $100 \mathrm{mM} \mathrm{KCl}, 10 \mathrm{mM}$ HEPES, $5 \mathrm{mM} \mathrm{KH}_{2} \mathrm{PO}_{4}$ and $\mathrm{pH} 7.2$ in the presence of $0.2 \%$ fatty acid free bovine serum albumin (BSA). The XFe96 plate was coated with polyethylenimine (1:15,000 dilution), and incubated overnight at $37^{\circ} \mathrm{C}$, and the polyethylenimine was removed in the assay day. $20 \mu \mathrm{L}$ of $0.5 \mathrm{mg} / \mathrm{mL}$ of isolated cardiac mitochondria were loaded into the XFe96 plate, and then mitochondria were centrifuged at $3000 \boldsymbol{g}$ for $7 \mathrm{~min}$ at https://joe.bioscientifica.com

https://doi.org/10.1530/JOE-18-0673 (c) 2019 Society for Endocrinology Published by Bioscientifica Ltd. Printed in Great Britain 
$4^{\circ} \mathrm{C}$. Then, the mitochondria were incubated for $30 \mathrm{~min}$ at $37^{\circ} \mathrm{C}$ before starting the assay. Firstly, pyruvate/malate (final concentration: $5 \mathrm{mM}$ each) was added as NADHlinked substrates, followed by ADP (final concentration: $1 \mathrm{mM}$ ) administration to determine mitochondrial state 3 respiration. State 4 respiration was measured after adding oligomycin (final concentration: $1 \mu \mathrm{M}$ ). The respiratory control ratio was calculated from state 3 /state 4 respiration and reported as cardiac mitochondrial function (Apaiajai et al. 2018).

\section{Western blot analysis}

The apex of the heart was used to carry out Western blot analysis. Sixty micrograms of the total protein from the tissue were mixed with a loading buffer, and the protein was loaded onto 10\% SDS-acrylamide gels and separated by electrophoresis. Then, the proteins were transferred onto a 45- $\mu \mathrm{m}$ nitrocellulose membrane (GE healthcare) in a wet-tank blotting system (Bio Rad). The membranes were blocked in 5\% skim milk or 5\% BSA in a Trisbuffered saline and Tween (TBST). The membranes were incubated overnight with primary antibodies including peroxisome proliferator-activated receptor (PGC- $1 \alpha$ ), carnitine palmitoyltransferase 1 (CPT-1), optic atrophy protein 1 (OPA1), phosphorylation of dynamin-related protein 1 at serine 616 (p-Drp1 $1^{\text {ser616) }}$ and dynamin-related protein 1 (Drp1). Then, the membranes were transferred to secondary antibodies. These membranes were exposed to an enhanced chemiluminescent substrate (Clarity Western ECL Substrate, Bio Rad). The Western blot pictures and densitometric analysis were carried out using the ChemiDoc Imaging system with Image Lab software (Bio Rad) (Apaijai et al. 2013).

\section{TUNEL assay}

Cardiac apoptosis was determined using TUNEL-positive cells (Roche). For in situ labeling, the mid-section of the cardiac tissue slices was placed in $1 \times$ PBS for $10 \mathrm{~min}$ after dehydration. The samples were covered with $50 \mu \mathrm{L}$ of Proteinase $\mathrm{k}$ solution (1:50) for $30 \mathrm{~min}$ followed by $50 \mu \mathrm{L}$ of Cytonin for $120 \mathrm{~min}$. For positive control, the samples were covered with TACS nuclease 1:50 in TACS nuclease buffer. TUNEL-positive cells were detected with a fluorescence microscope (Nikon) at $\lambda_{\text {ex }} 494 \mathrm{~nm}$ and $\lambda_{\text {em }}$ $512 \mathrm{~nm}$. DAPI was detected at $\lambda_{\mathrm{ex}} 358 \mathrm{~nm}$ and $\lambda_{\mathrm{em}} 461 \mathrm{~nm}$. The apoptosis index was calculated as a percentage of the number of TUNEL-positive apoptotic cells over the total number of nucleated cells (DAPI staining) (Nuntaphum et al. 2018).

\section{Statistical analysis}

Data are presented as mean \pm s.e. All statistical analyses were performed using GraphPad 6.0. Data were analyzed using a one-way ANOVA followed by Tukey's post-hoc test; $P<0.05$ was considered statistically significant.

\section{Results}

\section{Combined low-dose TRT with vildagliptin improved metabolic parameters in obese, insulin-resistant rats with testosterone deprivation}

In the NDOV group, ORX reduced both body and visceral fat weight, compared with those in NDS (Fig. 2A and B). Treatment with physiological dose TRT and combined low-dose TRT with vildagliptin, but not vildagliptin alone, increased body weight in NDOV rats, compared with NDS rats (Fig. 2A and B). These results indicated that this improvement in ND rats was due to testosterone, not vildagliptin.

In the HFS group, HFD rats had higher body and visceral fat weight than NDS rats (Fig. 2A and B). In the HFOV group, HFOV rats had lower body weight and visceral fat weight than HFS rats (Fig. 2A and B). Treatment with a physiological dose of TRT and combined lowdose TRT with vildagliptin, but not vildagliptin alone, increased body weight in HFO rats, compared with HFOV rats (Fig. 2A). However, all treatments did not affect visceral fat weight in both NDO and HFO rats, compared with NDOV and HFOV rats (Fig. 2B). Similar to ND rats, these results indicated that this improvement in HFO rats was due to testosterone, not vildagliptin.

In the HFD groups, plasma testosterone levels were not affected when compared with NDS rats (Fig. 2C). ORX effectively reduced plasma testosterone levels in both NDOV and HFOV rats, compared with the sham operation groups (Fig. 2C). Treatment with a physiological dose TRT and a combined low-dose TRT with vildagliptin, but not vildagliptin alone, restored plasma testosterone levels in both NDO and HFO rats, compared with NDOV and HFOV rats (Fig. 2C). These results indicated that the increased plasma testosterone in NDO and HFO rats was due to testosterone, not vildagliptin.

With regard to insulin resistance parameters in the HFD group, the HFD increased plasma insulin levels and 


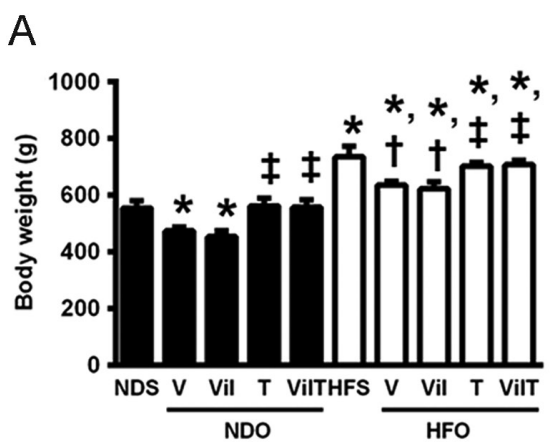

B
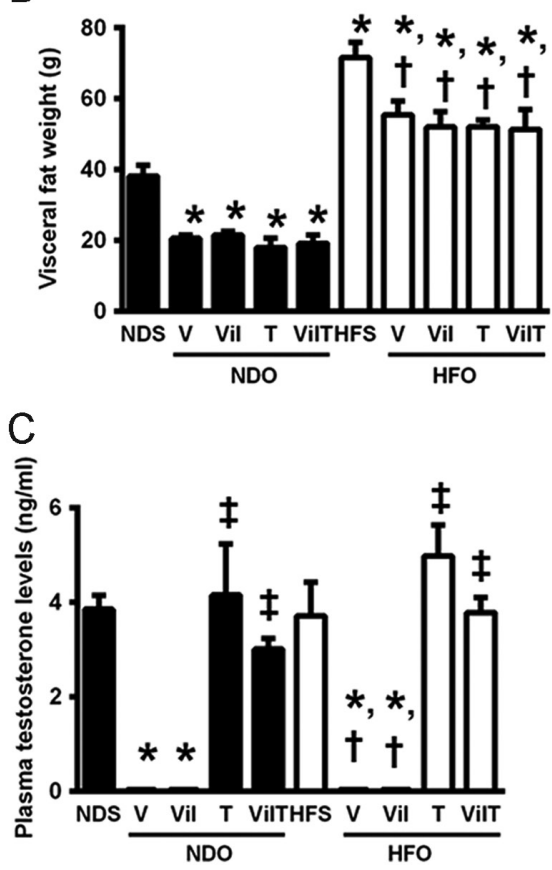

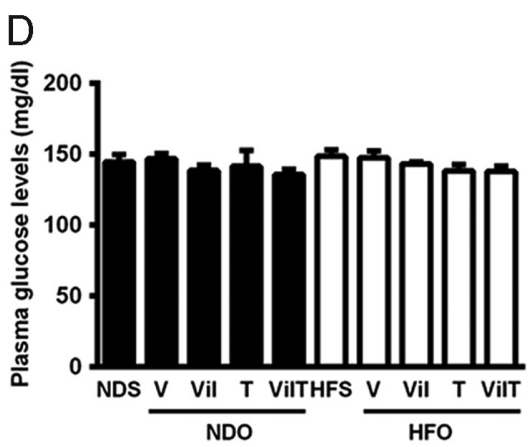

E

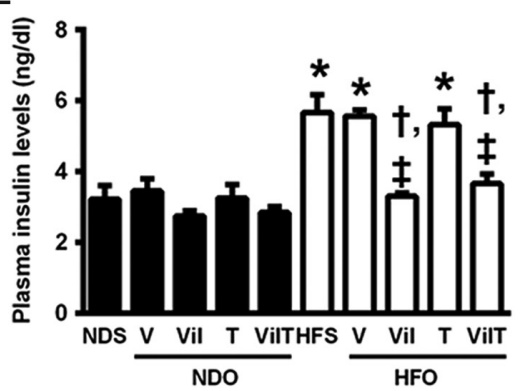

F

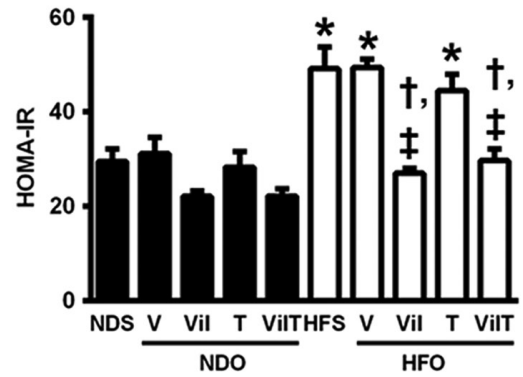

\section{Figure 2}

The effects of vildagliptin, a physiological dose TRT, and combined low-dose TRT with vildagliptin on metabolic parameters. (A) Body weight, (B) visceral fat weight, (C) plasma testosterone levels, (D) plasma glucose levels, (E) plasma insulin levels, (F) HOMA-IR index. ${ }^{*} P<0.05$ vs NDS, ${ }^{t} P<0.05$ vs HFS, $¥ P<0.05$ vs orchiectomized rats treated with vehicle on the same diet. HFO, high-fat diet rats with orchiectomy; HFS, high-fat diet rats with sham operation; HOMA, homeostatic model assessment; NDO, normal diet rats with orchiectomy; NDS, normal diet rats with sham operation; T, physiological dose testosterone replacement therapy; $\vee$, vehicle; Vil, vildagliptin; VilT, combined low-dose testosterone replacement therapy with vildagliptin.
HOMA-IR index without any changes in plasma glucose levels, compared with NDS rats (Fig. 2D, E and F). In the HFOV group, ORX did not affect plasma insulin levels, glucose levels or HOMA-IR index, when compared with HFS rats (Fig. 2D, E and F). Treatment with vildagliptin and combined low-dose TRT with vildagliptin, but not a physiological dose TRT, reduced plasma insulin levels and HOMA-IR index in HFO rats, compared with HFOV rats (Fig. 2D, E and F). These results indicated that an improved insulin-resistant status in HFO rats was due to vildagliptin, not testosterone.

With regard to lipid profiles in the HFD group, HFD increased plasma total cholesterol and LDL-C levels, compared with NDS rats (Fig. $3 \mathrm{~A}$ and D). In the HFOV group, ORX did not affect plasma lipid profiles, when compared with HFS rats (Fig. 3A and D). Treatment with vildagliptin, a physiological dose TRT and combined lowdose TRT with vildagliptin reduced both plasma total cholesterol and LDL-C levels in HFO rats, compared with HFOV rats (Fig. 3A and D). However, plasma triglyceride and HDL-C levels were no different between the groups (Fig. 3B and C). In the ND group, all lipid profile parameters were no different between groups (Fig. 3A, B, C and D). These results indicated that an improvement in plasma lipid profiles in HFO rats was due to both vildagliptin and testosterone.

\section{Combined low-dose TRT with vildagliptin improved LV} function and cardiac autonomic balance and reduced BP in obese, insulin-resistant rats with testosterone deprivation

In NDO rats the \%LV EF was decreased, whereas the LF/HF ratio, SBP and DBP were increased 12 weeks after ORX, compared with NDS rats (Fig. 4A, B, C and D). Treatment with vildagliptin, a physiological dose TRT 


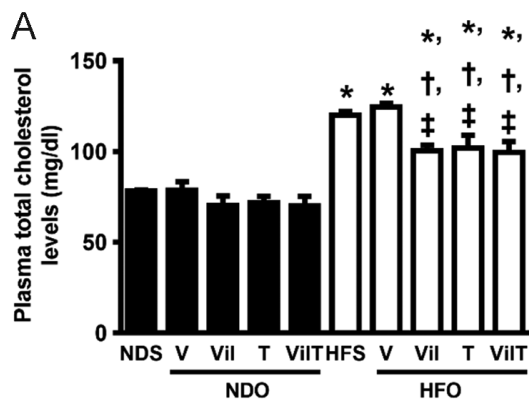

C

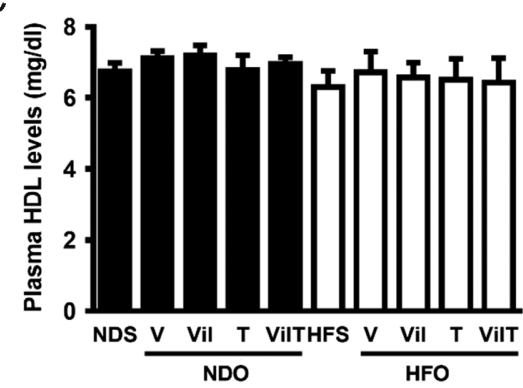

B

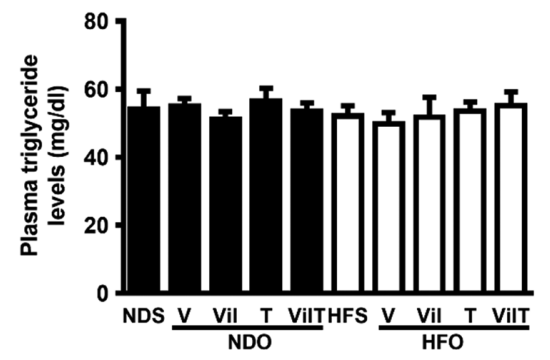

D

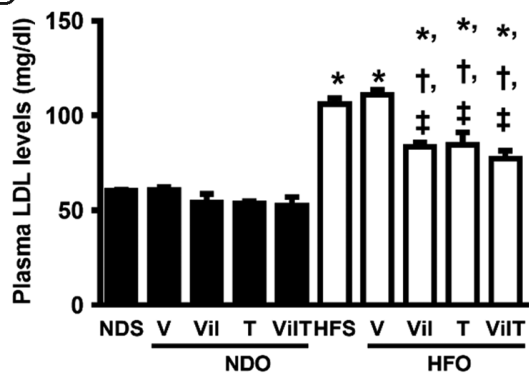

\section{Figure 3}

The effects of vildagliptin, a physiological dose TRT, and combined low-dose TRT with vildagliptin on plasma lipid profiles. (A) Plasma total cholesterol levels, (B) plasma triglyceride levels, (C) plasma HDL-C levels, (D) plasma LDL-C levels. $\star P<0.05$ vs NDS, ${ }^{\dagger} P<0.05$ vs HFS, ${ }^{\ddagger} P<0.05$ vs orchiectomized rats treated with vehicle on the same diet. HFO, high-fat diet rats with orchiectomy; HFS, high-fat diet rats with sham operation; NDO, normal diet rats with orchiectomy; NDS, normal diet rats with sham operation; T, physiological dose testosterone replacement therapy; V, vehicle; Vil, vildagliptin; VilT, combined low-dose testosterone replacement therapy with vildagliptin. and combined low-dose TRT with vildagliptin improved \%LVEF, LF/HF ratio, SBP, DBP and EDP, compared with NDOV rats (Fig. 4E, F, G and $\mathrm{H}$ ).

At week 12 after the operation, HFS rats had a decreased \%LV EF and an increased LF/HF ratio, SBP and DBP, compared with NDS rats (Fig. 4A, B and C). In the HFO group, \%LV EF markedly decreased, whereas the LF/HF ratio and SBP were markedly increased, compared with HFS rats (Fig. 4A, B and C). However, the DBP was no different between HFS and HFO rats (Fig. 4D).

After 4 weeks of treatment of the HFO rats, the vildagliptin, a physiological dose TRT and combined low-dose TRT with vildagliptin all led to an increased \%LV EF, compared with HFOV rats (Fig. 4E). However, all treatments decreased the LF/HF ratio, SBP and DBP to similar extents when compared with HFOV rats (Fig. 4F, $\mathrm{G}$ and $\mathrm{H})$.

Invasive LV function was determined and the data demonstrated that HR, ESP and $\mathrm{dP} / \mathrm{dt} \max$ were no different among groups (Fig. 5A, B and D). In the NDOV group, EDP and SV were decreased, compared with NDS rats (Fig. 5C and F). Treatment with vildagliptin, a physiological dose of TRT and combined low-dose TRT with vildagliptin improved EDP and SV in NDO rats, compared with NDOV rats (Fig. 5C and F). In the HFS group, EDP was increased, dP/dt min and SV were decreased, compared with NDS rats (Fig. 5C, E and F). In the HFOV group, EDP was markedly increased, $\mathrm{dP} / \mathrm{dt}$ min and SV were markedly decreased, compared with HFS rats (Fig. 5C, E and F). Treatment with vildagliptin, physiological dose of TRT, and combined low-dose TRT with vildagliptin improved $\mathrm{EDP}, \mathrm{dP} / \mathrm{dt}$ min and SV in $\mathrm{HFO}$ rats, compared with HFOV rats (Fig. 5C, E and F). These results indicated that the cardiac adverse effects could be attenuated by both vildagliptin and testosterone.

\section{Combined low-dose TRT with vildagliptin improved cardiac mitochondrial function in obese, insulin-resistant rats with testosterone deprivation}

In the NDOV group, ORX reduced the respiratory control ratio and increased mitochondrial ROS production and mitochondrial swelling, when compared with the NDS group (Fig. 6A, B and D). Treatment with vildagliptin, a physiological dose TRT and combined low-dose TRT with vildagliptin all improved the respiratory control ratio, mitochondrial ROS production and mitochondrial swelling, compared with NDOV rats (Fig. 6A, B and D). The representative pictures of cardiac mitochondria are shown in Fig. 6E. However, ORX did not affect mitochondrial membrane potential in ND rats (Fig. 6C).

In the HFS group, the respiratory control ratio was decreased, mitochondrial ROS production, mitochondrial membrane depolarization and mitochondrial swelling all being increased when compared with NDS rats (Fig. 6A, B, C and D). In the HFOV group, respiratory control ratio was markedly decreased, along with a marked increase in mitochondrial ROS production and mitochondrial swelling (Fig. 6A, $B$ and D), compared with HFS rats. Treatment with 
A

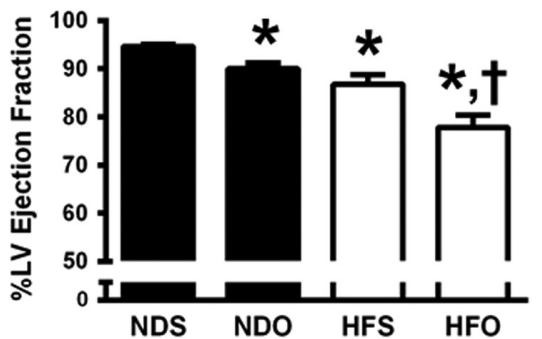

B

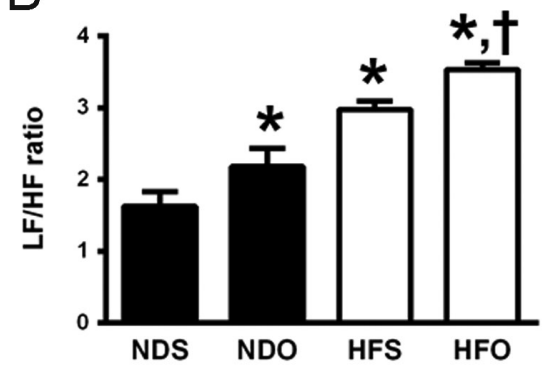

C
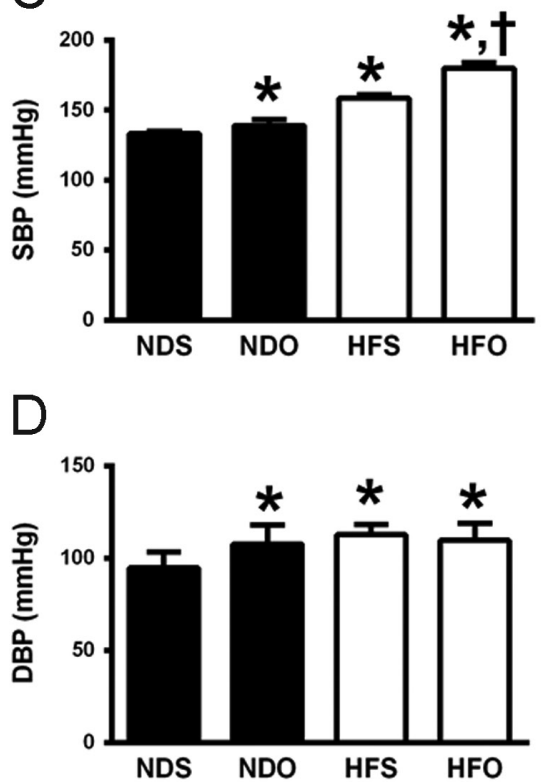

\section{E}

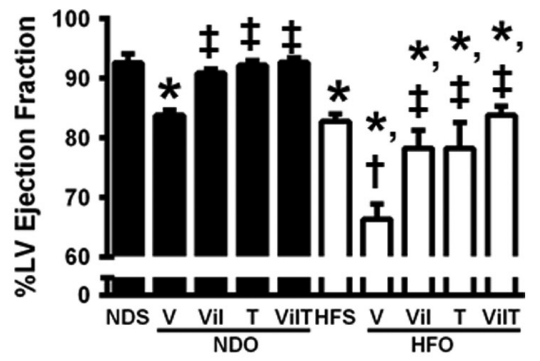

F

*,

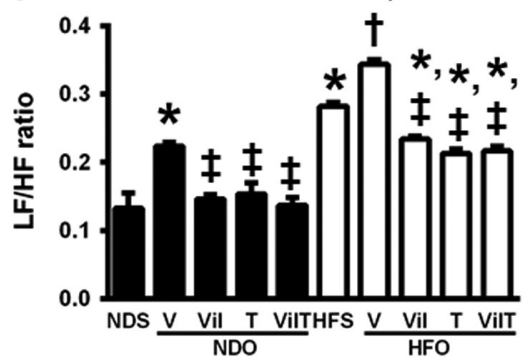

G

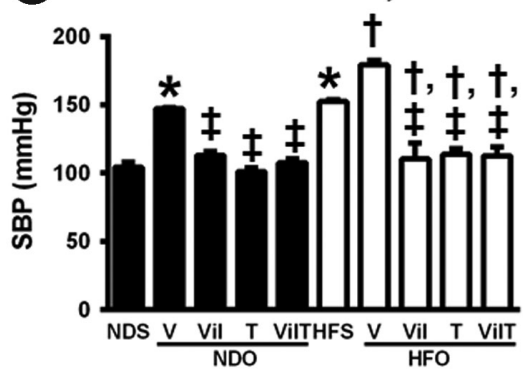

$\mathrm{H}$

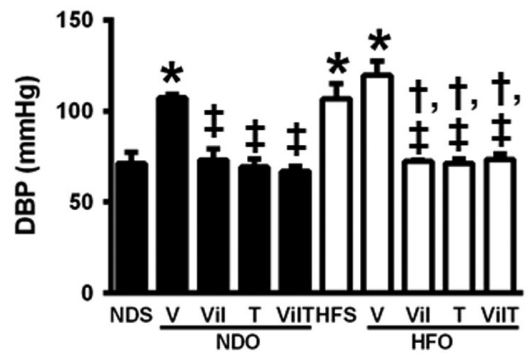

Figure 4

The effects of vildagliptin, a physiological dose TRT, and combined low-dose TRT with vildagliptin on LV function, cardiac autonomic function, and blood pressure. (A) \%LV EF prior to treatment, (B) $\mathrm{LF} / \mathrm{HF}$ ratio prior to treatment, (C) SBP prior to treatment, (D) DBP prior to treatment, (E) \%LV EF after treatment, (F) LF/HF ratio after treatment, (G) SBP after treatment, (H) DBP after treatment. $\star P<0.05$ vs NDS, ${ }^{\dagger} P<0.05$ vs HFS, $¥ P<0.05$ vs orchiectomized rats treated with vehicle on the same diet. DBP, diastolic blood pressure; HFO, high-fat diet rats with orchiectomy; HFS, high-fat diet rats with sham operation; NDO, normal diet rats with orchiectomy; NDS, normal diet rats with sham operation; LF/HF ratio, low-frequency/ high-frequency ratio; LV, left-ventricle; SBP, systolic blood pressure; $T$, physiological dose testosterone replacement therapy; $\mathrm{V}$, vehicle; Vil, vildagliptin; VilT, combined low-dose testosterone replacement therapy with vildagliptin. vildagliptin, a physiological dose TRT, and combined low-dose TRT with vildagliptin improved the respiratory control ratio and decreased mitochondrial ROS production, mitochondrial membrane depolarization and mitochondrial swelling, compared to HFOV rat data (Fig. 6A, B, C and D). The representative pictures of cardiac mitochondria are shown in Fig. 6E. These results indicated that these improvements were due to both vildagliptin and testosterone.
Combined low-dose TRT with vildagliptin improved cardiac mitochondrial biogenesis, mitochondrial dynamics and reduced cardiac apoptosis in obese, insulin-resistant rats with testosterone deprivation

In the NDOV group, ORX reduced PGC-1 $\alpha$, CPT-1, OPA1

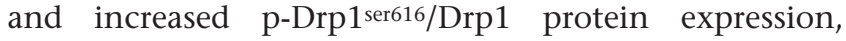
compared with NDS rats (Fig. 7A, B, C and D). Treatment with vildagliptin, a physiological dose TRT and combined 


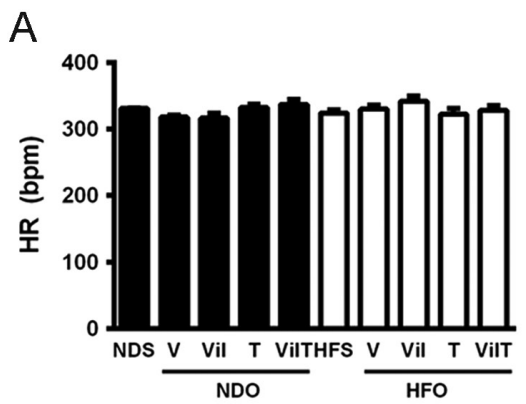

B

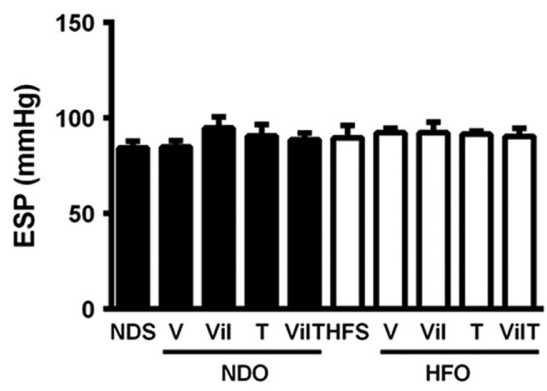

C

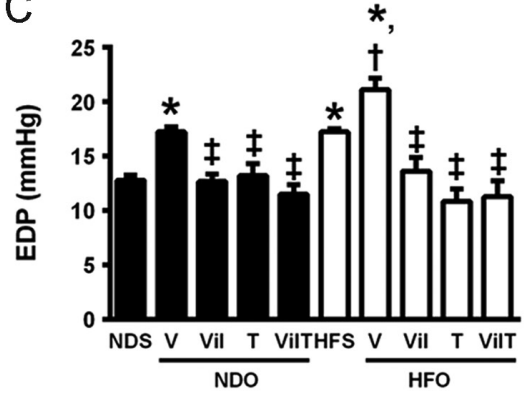

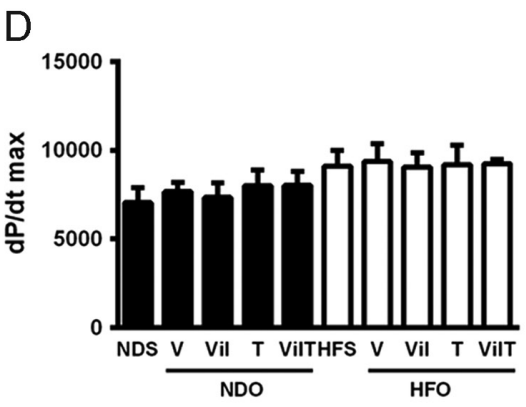

E

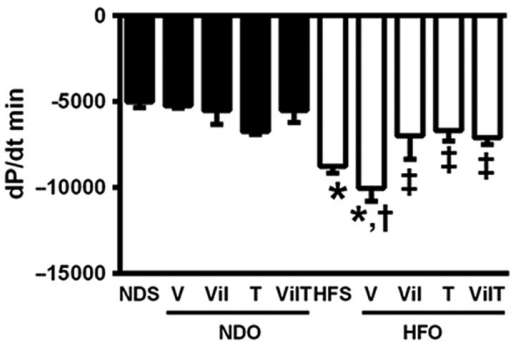

F

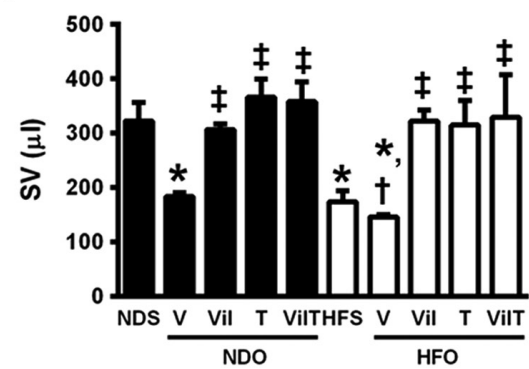

\section{Figure 5}

The effects of vildagliptin, a physiological dose TRT, and combined low-dose TRT with vildagliptin on invasive LV function parameters. (A) HR, (B) ESP, (C) EDP, (D) dP/dt max, (E) dP/dt min, (F) SV. $\star P<0.05$ vs NDS, ${ }^{\dagger} P<0.05$ vs HFS, ${ }^{*} P<0.05$ vs orchiectomized rats treated with vehicle on the same diet. EDP, end diastolic pressure; ESP, end-systolic pressure; HFO, high-fat diet rats with orchiectomy; HFS, high-fat diet rats with sham operation; HR, heart rate; NDO, normal diet rats with orchiectomy; NDS, normal diet rats with sham operation; SV, stroke volume; T,

physiological dose testosterone replacement therapy; $\mathrm{V}$, vehicle; Vil, vildagliptin; VilT, combined low-dose testosterone replacement therapy with vildagliptin. low-dose TRT with vildagliptin increased PGC-1 $\alpha$, CPT-1, OPA1 protein expression in NDO rats, compared with NDOV rats (Fig. 7A, B and D). In addition, treatment with a physiological dose TRT and combined lowdose TRT with vildagliptin, but not vildagliptin alone, reduced p-Drp1ser616/Drp1 protein expression in HFO rats, compared with HFOV rats (Fig. 7C).

In HFS rats, our Western blot data showed that HFD decreased PGC-1 $\alpha$, CPT-1, increased p-Drp1 1 ser616/Drp1 and reduced OPA1 protein expression, in comparison to NDS rats (Fig. 7A, B, C and D). In the HFOV group, ORX did not affect PGC-1 $\alpha$, CPT-1, p-Drp1ser616/Drp1 and OPA1 protein expression, compared with the results from the HFS group (Fig. 7A, B, C and D). Treatment with vildagliptin, a physiological dose TRT, and combined low-dose TRT with vildagliptin enhanced the expression of all proteins, PGC-1 $1 \alpha$, CPT-1 and OPA-1 in HFO rats, compared with HFOV rats (Fig. 7A, B and D). In addition, a physiological dose TRT and combined low-dose TRT with vildagliptin, but not vildagliptin alone, reduced p-Drp1ser616/Drp1 protein expression in HFO rats, compared with HFOV rats (Fig. 7C). These results indicated that vildagliptin alone could not reduce mitochondrial fission in both NDO and HFO rats.

As regards cardiac apoptosis, in ND rats, the higher TUNEL $^{+}$cells were found in NDOV rats than NDS rats (Fig. 8A and B). Treatment with vildagliptin, physiological dose TRT and combined low-dose TRT with vildagliptin reduced $\mathrm{TUNEL}^{+}$cells in NDO rats, compared to NDOV (Fig. 8A and $\mathrm{B}$ ). In the HFD group, HFS rats had higher numbers of TUNEL+ cells than NDS rats (Fig. 8A and B), and ORX markedly increased numbers of TUNEL ${ }^{+}$cells, compared with HFS rats (Fig. 8A and B). Treatment with vildagliptin, a physiological dose TRT and combined lowdose TRT with vildagliptin all led to a decrease in TUNEL ${ }^{+}$ cells in HFO rats, compared with HFOV (Fig. 8A and B). These results indicated that this improvement was due to both vildagliptin and testosterone. 
A

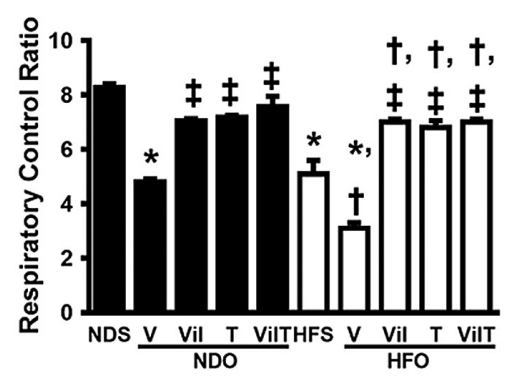

C

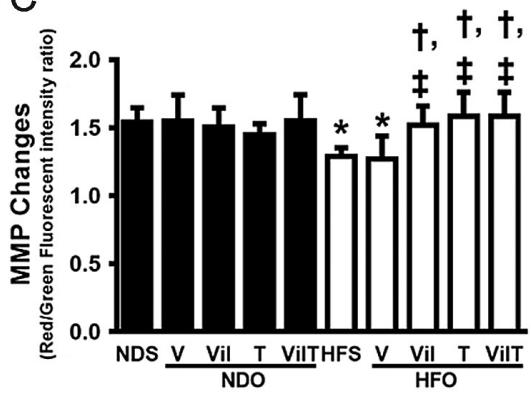

E

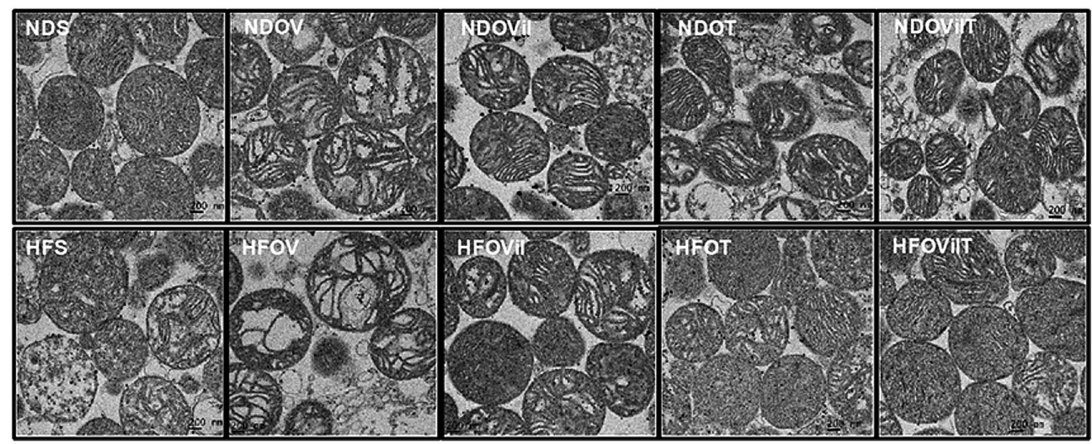

B

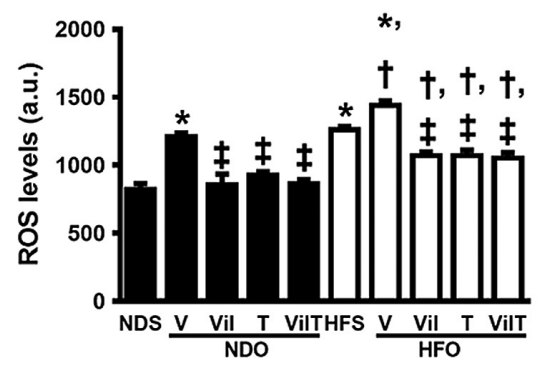

D

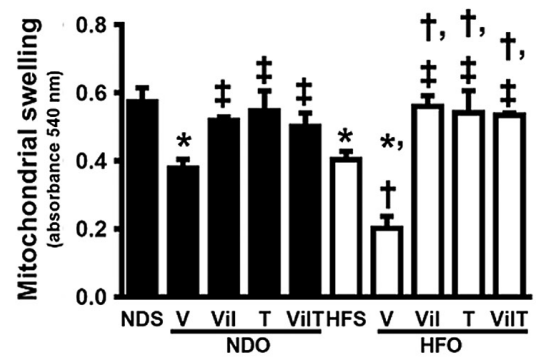

Figure 6

The effects of vildagliptin, a physiological dose TRT, and combined low-dose TRT with vildagliptin on cardiac mitochondrial function. (A) Respiratory control ratio, (B) mitochondrial ROS levels,

(C) mitochondrial membrane potential changes, (D) mitochondrial swelling, (E) representative pictures of cardiac mitochondria. ${ }^{*} P<0.05$ vs NDS, ${ }^{\dagger} P<0.05$ vs HFS, $\ddagger P<0.05$ vs orchiectomized rats treated with vehicle on the same diet. HFO, high-fat diet rats with orchiectomy; HFS, high-fat diet rats with sham operation; NDO, normal diet rats with orchiectomy; NDS, normal diet rats with sham operation; ROS, reactive oxygen species; $T$, physiological dose testosterone replacement therapy; V, vehicle; Vil, vildagliptin; VilT, combined low-dose testosterone replacement therapy with vildagliptin.

\section{Discussion}

The major findings of this study are as follows: (1) testosterone deprivation caused body weight loss in lean and obese insulin-resistant rats, but it did not aggravate metabolic impairments in obese, insulinresistant rats; (2) testosterone deprivation impaired LV function in lean rats, and it aggravated LV dysfunction in obese, insulin-resistant rats; (3) a physiological dose TRT and combined low-dose TRT with vildagliptin restored plasma testosterone levels and increased body weight in both lean and obese, insulin-resistant rats with testosterone deprivation; (4) vildagliptin and combined low-dose TRT with vildagliptin improved insulin-resistant status in obese, insulin-resistant rats with testosterone deprivation; (5) vildagliptin, a physiological dose TRT, and combined low-dose TRT with vildagliptin improved LV function to similar extents in both lean and obese, insulin-resistant rats with testosterone deprivation via the reduction of cardiac mitochondrial dysfunction and apoptosis, and increasing mitochondrial fusion; (6) physiological dose TRT and combined low-dose TRT with vildagliptin, but not vildagliptin alone, reduced mitochondrial fission in both lean and obese insulinresistant rats with testosterone deprivation.

Our previous studies reported that long-term HFD consumption induced obesity and metabolic impairments such as hyperinsulinemia and dyslipidemia (Pratchayasakul et al. 2011, Apaijai et al. 2012, 2013, Pintana etal.2015, Pongkan etal. 2016, Apaiajai etal.2018). Consistent with those studies, in this study, the HFD-fed rats developed obesity and insulin resistance as indicated by body weight gain, hyperinsulinemia and euglycemia. A review of clinical data shows that obese non-diabetic men have lower serum free testosterone levels (Dhindsa et al. 2010), and it is associated with cardiovascular risk (Cassimatis et al. 2016). Therefore, the bilateral ORX was done in both obese, insulin-resistant rats and 


\begin{tabular}{l|l|l|r|r|}
$\begin{array}{l}\text { Journal of } \\
\text { Endocrinology }\end{array}$ & A Arinno, N Apaijai et al. & $\begin{array}{l}\text { Testosterone and vildagliptin in } \\
\text { castrated rat }\end{array}$ & $\mathbf{2 4 0 : 3}$ & $\mathbf{4 7 7}$ \\
\hline
\end{tabular}

A

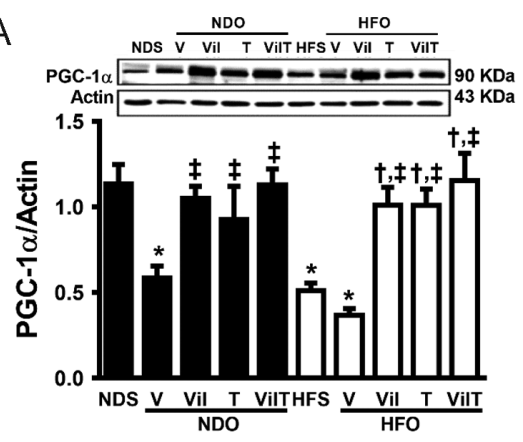

C
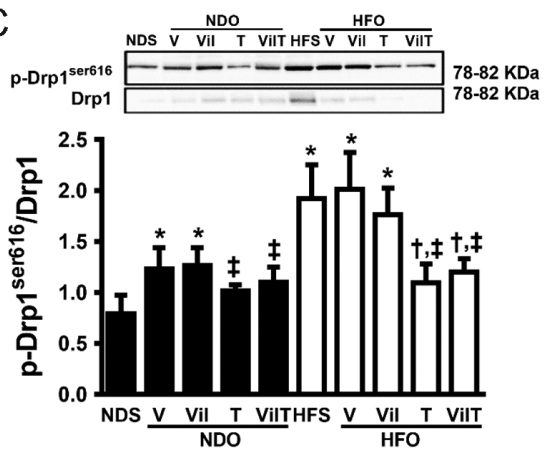

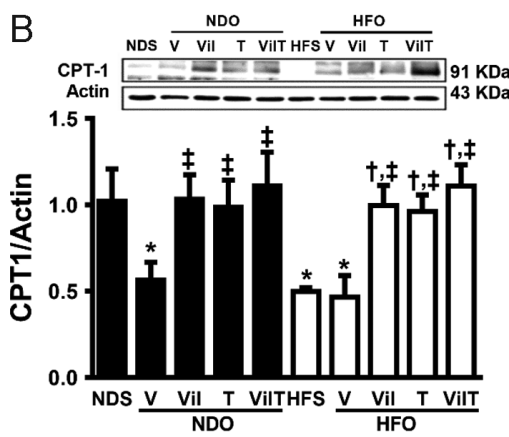

D
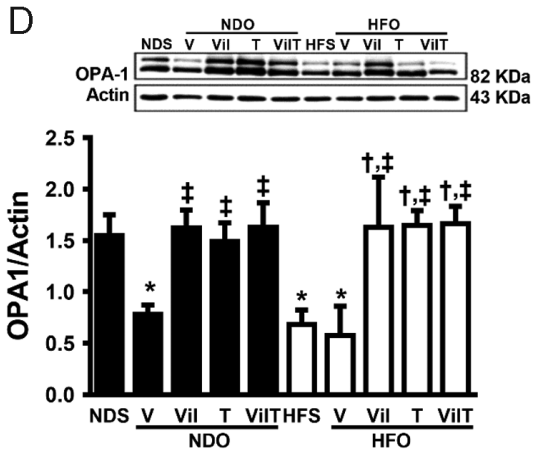

Figure 7

The effects of vildagliptin, a physiological dose TRT, and combined low-dose TRT with vildagliptin on cardiac mitochondrial biogenesis and dynamics. (A) PGC-1 $\alpha$ protein expression, (B) CPT-1 protein expression, (C) p-Drp1 ser616/Drp1 protein expression, (D) OPA-1 protein expression. $* P<0.05$ vs NDS, ${ }^{\dagger} P<0.05$ vs HFS, ${ }^{\ddagger} P<0.05$ vs orchiectomized rats treated with vehicle on the same diet. CPT-1, carnitine palmitoyl transferase 1; Drp1, dynamin-related protein 1; HFO, high-fat diet rats with orchiectomy; HFS, high-fat diet rats with sham operation; NDO, normal diet rats with orchiectomy; NDS, normal diet rats with sham operation; OPA-1, optic atrophy protein 1; PGC-1 $\alpha$, peroxisome proliferated receptor 1 gamma; T, physiological dose testosterone replacement therapy; $\mathrm{V}$, vehicle; Vil, vildagliptin; VilT, combined low-dose testosterone replacement therapy with vildagliptin. lean rats to represent the pathology of cardiometabolic impairments. Although data from clinical study reported that obesity is strongly associated with testosterone deprivation (Eriksson et al. 2017), plasma testosterone level was not decreased in our obese, insulin-resistant rats. This inconsistent finding could be due to the short duration of HFD feeding used (28 weeks) in our study. This is supported by previous reports in which study by
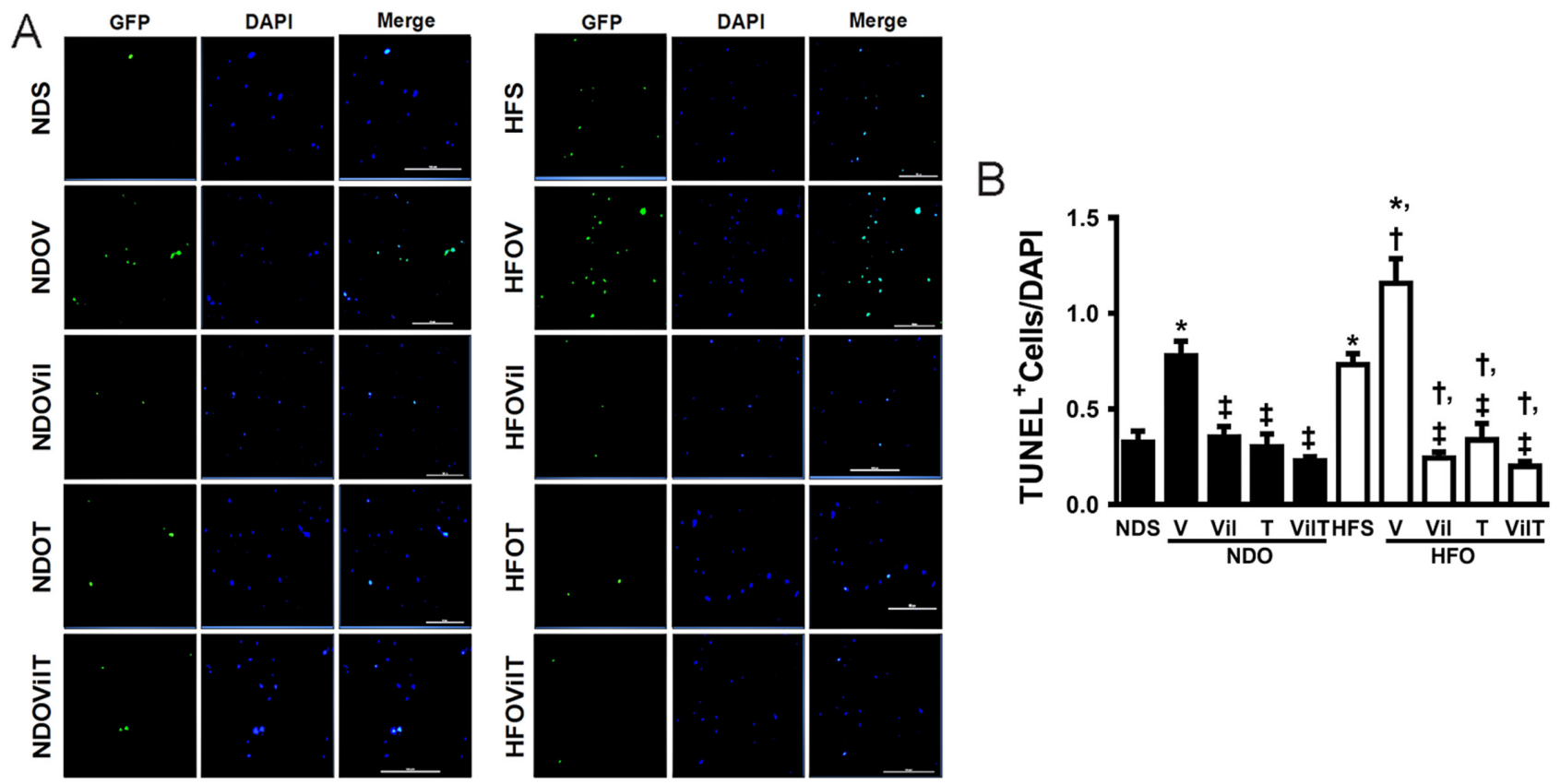

Figure 8

The effects of vildagliptin, a physiological dose TRT, and combined low-dose TRT with vildagliptin on cardiac apoptosis. (A) Representative pictures of TUNEL, (B) TUNEL ${ }^{+}$cells/DAPI. * $P<0.05$ vs NDS, ${ }^{\circ} P<0.05$ vs HFS, $¥ P<0.05$ vs orchiectomized rats treated with vehicle on the same diet. HFO, high-fat diet rats with orchiectomy; HFS, high-fat diet rats with sham operation; NDO, normal diet rats with orchiectomy; NDS, normal diet rats with sham operation; $\mathrm{T}$, physiological dose testosterone replacement therapy; $\mathrm{V}$, vehicle; Vil, vildagliptin; VilT, combined low-dose testosterone replacement therapy with vildagliptin. 
Donner and colleagues reported that the rats fed with the obesogenic diet for 28 weeks had a similar level of serum testosterone level as the control rats (Donner et al. 2015), whereas Silva and colleagues reported that HFD feeding for 30 weeks effectively reduced plasma testosterone levels in obese rats, compared with lean rats (Silva et al. 2015). Our data indicated that testosterone deprivation did not affect peripheral insulin-resistant status, but it led to a reduction in both body weight and visceral fat weight. Testosterone plays a role in regulating food intake via increasing neuropeptide $\mathrm{Y}$ (NPY), and the levels of NPY were reduced in median eminence, arcuate nucleus and ventromedial nucleus of the brain after ORX (Sahu et al. 1989). The reduction of NPY caused a reduction in food intake (Pintana et al. 2015, Pongkan et al. 2016, Apaiajai et al. 2018), leading to a reduction in body weight and visceral fat weight. The physiological level of serum/ plasma testosterone in rats is $1.5-2.9 \mathrm{ng} / \mathrm{mL}$ (Pintana et al. 2015, Pongkan et al. 2016). The physiological dose of testosterone administered to the rats in this study was selected based upon the data from our previous studies (Pintana et al. 2015, Pongkan et al. 2015, 2016). We reported that testosterone, at $2 \mathrm{mg} / \mathrm{kg}$ via s.c. injection for 8 weeks, effectively restored serum/plasma testosterone levels back to its physiological levels, and therefore was considered as a physiological dose of TRT. However, TRT has been reported to be associated with cardiovascular risk factors in the clinical setting such as impaired lipoprotein patterns, and it is related with an ischemic heart disease (Barrett-Connor 1995, Sinha-Hinkin et al. 2006, Fink et al. 2018). Thus, a low-dose TRT was used in this study. Our results suggested that the combined low-dose TRT with vildagliptin provided a similar cardioprotective effects as a physiological dose, which might help to reduce the future side effects of TRT in testosterone-deprived subjects. This is the first study to show the beneficial effects of a combination of low-dose TRT and vildagliptin on insulinresistant status, lipid profiles and LV function in obese subjects with testosterone deprivation.

Treatment with a physiological dose of TRT and the combined drugs increased plasma testosterone levels and body weight in lean and obese insulin-resistant rats with testosterone deprivation. TRT has been shown to increase the fat free mass in men with hypogonadism (Bhasin et al. 1997), and our results were consistent with this. However, although TRT increased body weight, the therapy did not affect visceral fat weight in both lean and obese, insulinresistant rats. These data suggested that TRT at both the physiological and low-dose increased body weight by increasing the fat free mass in lean and obese, insulinresistant rats with testosterone deprivation.

Regarding the insulin-resistant status, only vildagliptin and the combined drugs reduced insulin resistance in obese, insulin-resistant rats. Vildagliptin mainly inhibits DPP4 action, and prolongs the life span of glucagon like peptide 1 (Dei Cas et al. 2017). In addition it has been reported that vildagliptin is an antioxidant, and it helps to increase insulin receptor function, leading to an improved insulin sensitivity in obese, insulinresistant rats (Apaijai et al. 2013, Abdelsalam \& Safar 2015, Pintana et al. 2015). Our results clearly demonstrated that testosterone alone could not improve the peripheral insulin-resistant condition in this model.

When considering the lipid profiles, our data demonstrated that obese, insulin-resistant rats had high levels of both total and LDL-C cholesterol; however, testosterone deprivation did not affect the lipid profiles. All pharmacological interventions reduced plasma total cholesterol levels and LDL-C levels in similar proportions in obese, insulin-resistant rats with testosterone deprivation. Moreover, our data showed that the reduction in plasma lipid profiles was associated with an increased CPT-1 protein expression. CPT-1 is a rate-limiting enzyme that controls fatty acid transport, which plays an important role in regulating lipid metabolism in several organs including the heart (Kerner \& Hoppel 2000, Longo et al. 2016). It has been shown that the expression of CPT-1 was decreased in animals with metabolic syndrome (Schreurs et al. 2010, Shen et al. 2015). Moreover, the reduction of CPT-1 protein expression was found to be associated with the impairment of lipid metabolism, and increased plasma lipid profiles including cholesterol and triglyceride levels (Schreurs et al. 2010, Shen et al. 2015). Therefore, our data suggested that TRT and vildagliptin enhanced the expression of CPT-1, leading to improved lipid metabolism, and reduced plasma total cholesterol and LDL-C levels.

Although testosterone deprivation did not aggravate metabolic impairment, our results showed that it worsened LV dysfunction and cardiac autonomic imbalance, and blood pressure was markedly increased in obese, insulinresistant rats with testosterone deprivation, compared with intact obese, insulin-resistant rats. All pharmacological interventions effectively attenuated LV dysfunction, cardiac autonomic imbalance, and led to reduced blood pressure in both lean and obese, insulin-resistant rats with testosterone deprivation. We previously demonstrated that cardiac mitochondrial dysfunction including https://joe.bioscientifica.com

https://doi.org/10.1530/JOE-18-0673 (c) 2019 Society for Endocrinology Published by Bioscientifica Ltd.
Printed in Great Britain 
increased ROS production, mitochondrial depolarization and mitochondrial swelling is responsible for a worsening of LV dysfunction in obese, insulin-resistant rats with testosterone deprivation (Apaiajai et al. 2018). In this study, our data showed that all pharmacological interventions effectively attenuated cardiac mitochondrial dysfunction as indicated by decreasing cardiac mitochondrial oxidative stress, mitochondrial membrane depolarization and mitochondrial swelling, leading to improved mitochondrial respiration. An improvement in cardiac mitochondrial respiration is associated with an increase in mitochondrial ATP production (Brand \& Nicholls 2011), resulting in improving LV function in lean and obese, insulin-resistant rats with testosterone deprivation as observed in this study.

Mitochondria are dynamic organelles, their dynamics being regulated by the mitochondrial fusion and fission process. Mitochondrial fission is controlled by Drp1, which plays a role in generating the inter-connected mitochondrial network or promoting mitochondrial fragmentation (Lennon \& Salgia 2014, Dorn et al. 2015). Mitochondrial fusion is driven by mitofusin 1,2 (Mfn1,2) and optic atrophy type 1 (OPA-1) (Dorn et al. 2015). After Mfn1,2 are activated, they move from the outer to the inner membrane, and join with the OPA-1 enzyme to initiate mitochondrial fusion (Dorn et al.
2015). Thus, OPA-1 is an important protein during the fusion process as it is instrumental in the final step of mitochondrial fusion (Song et al. 2009). Our data showed that mitochondrial fission was markedly increased, and mitochondrial fusion was markedly decreased in obese, insulin-resistant rats with testosterone deprivation, compared with those which showed obese insulin resistance alone. On studying the mitochondrial fission results, a physiological dose of TRT and combined drugs could reduce mitochondrial fission, whereas vildagliptin alone failed to reduce mitochondrial fission in lean and obese, insulin-resistant rats with testosterone deprivation. In the case of mitochondrial fusion, all pharmacological interventions increased OPA- 1 protein expression, and led to increased mitochondrial fusion in lean and obese, insulin-resistant rats with testosterone deprivation. Thus, our Western blot data suggested that TRT plays more a significant role than vildagliptin in improving cardiac mitochondrial dynamics in lean and obese, insulinresistant rats with testosterone deprivation.

In addition, mitochondrial dysfunction is related to the induction of cardiac apoptosis. This occurs due to the release of cytochrome $c$ from the inner membrane of the mitochondria inducing caspase activation, finally leading to the fragmentation of DNA, a process detected by TUNEL (Wang \& Youle 2009). Our TUNEL data demonstrated
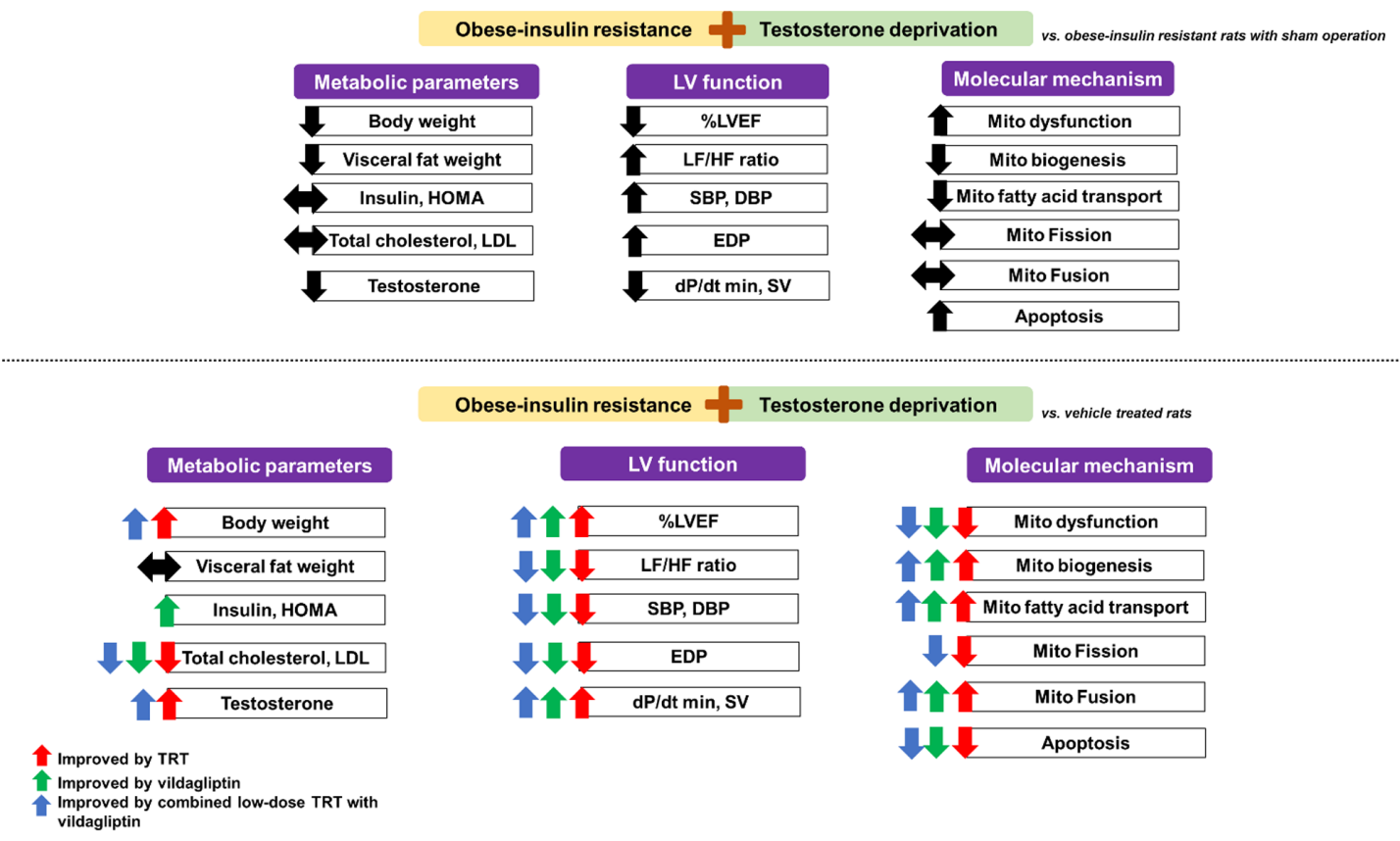

Obese-insulin resistance thestosterone deprivation

LV function

11 \%LVEF

LF/HF ratio

SBP, DBP

$\checkmark \sqrt{1}$ EDP

11 $\mathrm{dP} / \mathrm{dt} \min , \mathrm{SV}$
Molecular mechanism

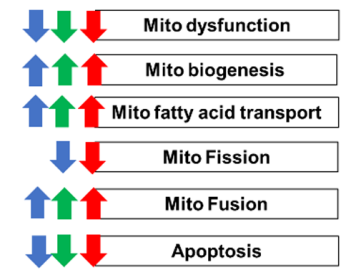

Figure 9

Summarized diagram of the effects of vildagliptin, physiological dose TRT, and combined low-dose TRT with vildagliptin on metabolic parameters, LV function, and mitochondrial function in obese, insulin-resistant rats with testosterone deprivation. The complete colored version of this figure is available at https://doi.org/10.1530/JOE-18-0673. 
that cardiac apoptosis was increased in obese, insulinresistant rats, and it was markedly increased in obese, insulin-resistant rats with testosterone deprivation. All pharmacological interventions improved cardiac mitochondrial function to a similar degree, leading to a reduction in cardiac apoptosis. This could also be responsible for an improvement in LV function (Fig. 9).

In this study, NDO rats had no changes in glucose levels, lipid profiles and insulin resistance. This is consistent with previous reports (Pintana et al. 2015, Pongkan et al. 2016). In ND rats, orchiectomy led to testosterone deprivation, and directly affected the mitochondria by increasing mitochondrial oxidative stress. An increasing of mitochondrial oxidative stress impaired the mitochondrial oxidative phosphorylation process (Murphy 2009, Kudryavtseva et al. 2016), leading to ATP depletion as shown by decreased respiratory control ratio in our NDO rats. Furthermore, mitochondrial oxidative stress disrupted the balance of mitochondrial dynamics, resulting in myocardial apoptosis. Therefore, both ATP depletion and myocardial apoptosis could lead to LV dysfunction in ND rats, and this effect was independent to metabolic disturbances.

In conclusion, the combined low-dose TRT with vildagliptin therapy effectively improved LV function to a similar extent to the physiological dose TRT alone or vildagliptin alone via improving mitochondrial fusion and reducing mitochondrial dysfunction and apoptosis in NDO and HFO rats. However, combined low-dose TRT with vildagliptin and the physiological dose TRT exerted greater benefit on the heart than vildagliptin alone via decreasing mitochondrial fission in both NDO and HFO rats. Our findings suggest that low-dose TRT combined with vildagliptin may be an alternative for physiological dose TRT in condition of obese, insulin resistance with testosterone deprivation, thus reducing the dose of testosterone to be used in this condition.

\section{Declaration of interest}

The authors declare that there is no conflict of interest that could be perceived as prejudicing the impartiality of the research reported.

\section{Funding}

This work was supported by the Thailand Research Fund TRF-Senior Research Scholar RTA6080003 (S C C), RSA6180071 (W P), TRG6080005 (N A); The National Research Council of Thailand (S C); a NSTDA Research Chair Grant from the National Science and Technology Development Agency Thailand (N C) and a Chiang Mai University Excellent Center Award (N C).

\section{Author contribution statement}

S C C and N C: Conception; A A, N A, P K, W P, TJ, S K: Performed experiments; A A, N A, S C C, N C: Analyzed the data; A A, N A, S C C, N C: Drafting the manuscript, revision of the manuscript and final approval.

\section{References}

Abdelsalam RM \& Safar MM 2015 Neuroprotective effects of vildagliptin in rat rotenone Parkinson's disease model: role of RAGE-NFkappaB and Nrf2-antioxidant signaling pathways. Journal of Neurochemistry 133 700-707. (https://doi.org/10.1111/jnc.13087)

Apaiajai N, Chunchai T, Jaiwongkam T, Kerdphoo S, Chattipakorn SC \& Chattipakorn N 2018 Testosterone deprivation aggravates leftventricular dysfunction in male obese insulin-resistant rats via impairing cardiac mitochondrial function and dynamics proteins. Gerontology 64 333-343. (https://doi.org/10.1159/000487188)

Apaijai N, Pintana H, Chattipakorn SC \& Chattipakorn N 2012 Cardioprotective effects of metformin and vildagliptin in adult rats with insulin resistance induced by a high-fat diet. Endocrinology 153 3878-3885. (https://doi.org/10.1210/en.2012-1262)

Apaijai N, Pintana H, Chattipakorn SC \& Chattipakorn N 2013 Effects of vildagliptin versus sitagliptin, on cardiac function, heart rate variability and mitochondrial function in obese insulin-resistant rats. British Journal of Pharmacology 169 1048-1057. (https://doi. org/10.1111/bph.12176)

Bae WJ, Zhu GQ, Choi SW, Jeong HC, Bashraheel F, Kim KS, Kim SJ, Cho HJ, Ha US, Hong SH, et al. 2017 Antioxidant and antifibrotic effect of a herbal formulation in vitro and in the experimental andropause via $\mathrm{Nrf} 2 / \mathrm{HO}-1$ signaling pathway. Oxidative Medicine and Cellular Longevity 2017 6024839. (https://doi. org/10.1155/2017/6024839)

Barrett-Connor EL 1995 Testosterone and risk factors for cardiovascular disease in men. Diabetes and Metabolism 21 156-161.

Bhasin S, Storer TW, Berman N, Yarasheski KE, Clevenger B, Phillips J, Lee WP, Bunnell TJ \& Casaburi R 1997 Testosterone replacement increases fat-free mass and muscle size in hypogonadal men. Journal of Clinical Endocrinology and Metabolism 82 407-413. (https://doi. org/10.1210/jcem.82.2.3733)

Bhasin S, Brito JP, Cunningham GR, Hayes FJ, Hodis HN, Matsumoto AM, Snyder PJ, Swerdloff RS, Wu FC \& Yialamas MA 2018 Testosterone therapy in men with hypogonadism: an Endocrine Society clinical practice guideline. Journal of Clinical Endocrinology and Metabolism 103 1715-1744. (https://doi.org/10.1210/jc.2018-00229)

Brand MD \& Nicholls DG 2011 Assessing mitochondrial dysfunction in cells. Biochemical Journal 435 297-312. (https://doi.org/10.1042/ BJ20110162)

Byrne M \& Nieschlag E 2003 Testosterone replacement therapy in male hypogonadism. Journal of Endocrinological Investigation 26 481-489. (https://doi.org/10.1007/BF03345206)

Cassimatis DC, Crim MT \& Wenger NK 2016 Low testosterone in men with cardiovascular disease or risk factors: to treat or not to treat? Current Treatment Options in Cardiovascular Medicine 18 75. (https:// doi.org/10.1007/s11936-016-0496-0)

Chen Y, Fu L, Han Y, Teng Y, Sun J, Xie R \& Cao J 2012 Testosterone replacement therapy promotes angiogenesis after acute myocardial infarction by enhancing expression of cytokines HIF-1a, SDF-1a and VEGF. European Journal of Pharmacology 684 116-124. (https://doi. org/10.1016/j.ejphar.2012.03.032)

Dei Cas A, Spigoni V, Cito M, Aldigeri R, Ridolfi V, Marchesi E, Marina M, Derlindati E, Aloe R, Bonadonna RC, et al. 2017 Vildagliptin, but not glibenclamide, increases circulating endothelial progenitor cell number: a 12-month randomized controlled trial in patients with type 2 diabetes. Cardiovascular Diabetology 16 27. (https://doi. org/10.1186/s12933-017-0503-0) https://joe.bioscientifica.com

https://doi.org/10.1530/JOE-18-0673
(C) 2019 Society for Endocrinology Published by Bioscientifica Ltd. Printed in Great Britain 
Dhindsa S, Miller MG, McWhirter CL, Mager DE, Ghanim H, Chaudhuri A \& Dandona P 2010 Testosterone concentrations in diabetic and nondiabetic obese men. Diabetes Care 33 1186-1192. (https://doi.org/10.2337/dc09-1649)

Donner DG, Elliott GE, Beck BR, Bulmer AC \& Du Toit EF 2015 Impact of diet-induced obesity and testosterone deficiency on the cardiovascular system: a novel rodent model representative of males with testosterone-deficient metabolic syndrome (TDMetS). PLoS ONE 10 e0138019. (https://doi.org/10.1371/journal.pone.0138019)

Dorn GW 2nd, Vega RB \& Kelly DP 2015 Mitochondrial biogenesis and dynamics in the developing and diseased heart. Genes and Development 29 1981-1991. (https://doi.org/10.1101/gad.269894.115)

Eleawa SM, Sakr HF, Hussein AM, Assiri AS, Bayoumy NM \& Alkhateeb M 2013 Effect of testosterone replacement therapy on cardiac performance and oxidative stress in orchidectomized rats. Acta Physiologica 209 136-147. (https://doi.org/10.1111/apha.12158)

Eriksson J, Haring R, Grarup N, Vandenput L, Wallaschofski H, Lorentzen E, Hansen T, Mellström D, Pedersen O, Nauck M, et al. 2017 Causal relationship between obesity and serum testosterone status in men: a bi-directional Mendelian randomization analysis PLOS ONE 12 e0176277. (https://doi.org/10.1371/journal. pone.0176277)

Fink J, Matsumoto M \& Tamura Y 2018 Potential application of testosterone replacement therapy as treatment for obesity and type 2 diabetes in men. Steroids 138 161-166. (https://doi.org/10.1016/j. steroids.2018.08.002)

Finkle WD, Greenland S, Ridgeway GK, Adams JL, Frasco MA, Cook MB, Fraumeni JF, Jr \& Hoover RN 2014 Increased risk of non-fatal myocardial infarction following testosterone therapy prescription in men. PLOS ONE 9 e85805. (https://doi.org/10.1371/journal. pone.0085805)

Hruby A \& Hu FB 2015 The epidemiology of obesity: a big picture. Pharmacoeconomics 33 673-689. (https://doi.org/10.1007/s40273-0140243-x)

Kerner J \& Hoppel C 2000 Fatty acid import into mitochondria. Biochimica et Biophysica Acta 1486 1-17. (https://doi.org/10.1016/ S1388-1981(00)00044-5)

Kudryavtseva AV, Krasnov GS, Dmitriev AA, Alekseev BY, Kardymon OL, Sadritdinova AF, Fedorova MS, Pokrovsky AV, Melnikova NV, Kaprin AD, et al. 2016 Mitochondrial dysfunction and oxidative stress in aging and cancer. Oncotarget 7 44879-44905. (https://doi. org/10.18632/oncotarget.9821)

Lennon FE \& Salgia R 2014 Mitochondrial dynamics: biology and therapy in lung cancer. Expert Opinion on Investigational Drugs 23 675-692. (https://doi.org/10.1517/13543784.2014.899350)

Longo N, Frigeni M \& Pasquali M 2016 Carnitine transport and fatty acid oxidation. Biochimica et Biophysica Acta 1863 2422-2435. (https://doi. org/10.1016/j.bbamcr.2016.01.023)

Murphy MP 2009 How mitochondria produce reactive oxygen species. Biochemical Journal 417 1-13. (https://doi.org/10.1042/BJ20081386)

Nuntaphum W, Pongkan W, Wongjaikam S, Thummasorn S, Tanajak P, Khamseekaew J, Intachai K, Chattipakorn SC, Chattipakorn N \& Shinlapawittayatorn K 2018 Vagus nerve stimulation exerts cardioprotection against myocardial ischemia/reperfusion injury predominantly through its efferent vagal fibers. Basic Research in Cardiology 113 22. (https://doi.org/10.1007/s00395-018-0683-0)

Pintana H, Pongkan W, Pratchayasakul W, Chattipakorn N \& Chattipakorn SC 2015 Dipeptidyl peptidase 4 inhibitor improves brain insulin sensitivity, but fails to prevent cognitive impairment in orchiectomy obese rats. Journal of Endocrinology 226 M1-M11. (https://doi.org/10.1530/JOE-15-0099)

Pongkan W, Chattipakorn SC \& Chattipakorn N 2015 Chronic testosterone replacement exerts cardioprotection against cardiac ischemia-reperfusion injury by attenuating mitochondrial dysfunction in testosterone-deprived rats. PLOS ONE 10 e0122503. (https://doi.org/10.1371/journal.pone.0122503)

Pongkan W, Pintana H, Jaiwongkam T, Kredphoo S, Sivasinprasasn S, Chattipakorn SC \& Chattipakorn N 2016 Vildagliptin reduces cardiac ischemic-reperfusion injury in obese orchiectomized rats. Journal of Endocrinology 231 81-95. (https://doi.org/10.1530/JOE-16-0232)

Pratchayasakul W, Kerdphoo S, Petsophonsakul P, Pongchaidecha A, Chattipakorn N \& Chattipakorn SC 2011 Effects of high-fat diet on insulin receptor function in rat hippocampus and the level of neuronal corticosterone. Life Sciences 88 619-627. (https://doi. org/10.1016/j.lfs.2011.02.003)

Reilly JJ, El-Hamdouchi A, Diouf A, Monyeki A \& Somda SA 2018 Determining the worldwide prevalence of obesity. Lancet 391 1773-1774. (https://doi.org/10.1016/S0140-6736(18)30794-3)

Sahu A, Kalra SP, Crowley WR \& Kalra PS 1989 Testosterone raises neuropeptide-Y concentration in selected hypothalamic sites and in vitro release from the medial basal hypothalamus of castrated male rats. Endocrinology 124 410-414. (https://doi.org/10.1210/endo-124$1-410)$

Schreurs M, Kuipers F \& van der Leij FR 2010 Regulatory enzymes of mitochondrial beta-oxidation as targets for treatment of the metabolic syndrome. Obesity Reviews 11 380-388. (https://doi. org/10.1111/j.1467-789X.2009.00642.x)

Shen Y, Xu X, Yue K \& Xu G 2015 Effect of different exercise protocols on metabolic profiles and fatty acid metabolism in skeletal muscle in high-fat diet-fed rats. Obesity 23 1000-1006. (https://doi.org/10.1002/ oby.21056)

Silva SA, Gobbo MG, Pinto-Fochi ME, Rafacho A, Taboga SR, Almeida EA, Góes RM \& Ribeiro DL 2015 Prostate hyperplasia caused by long-term obesity is characterized by high deposition of extracellular matrix and increased content of MMP-9 and VEGF. International Journal of Experimental Pathology 96 21-30. (https://doi.org/10.1111/iep.12107)

Sinha-Hinkin I, Cornford M, Gaytan H, Lee ML \& Bhasin S 2006 Effects of testosterone supplementation on skeletal muscle fiber hypertrophy and satellite cells in community-dwelling older men. Journal of Clinical Endocrinology and Metabolism 91 3024-3033. (https://doi. org/10.1210/jc.2006-0357)

Song Z, Ghochani M, McCaffery JM, Frey TG \& Chan DC 2009 Mitofusins and OPA1 mediate sequential steps in mitochondrial membrane fusion. Molecular Biology of the Cell 20 3525-3532. (https:// doi.org/10.1091/mbc.e09-03-0252)

Wang C \& Youle RJ 2009 The role of mitochondria in apoptosis. Annual Review of Genetics 43 95-118. (https://doi.org/10.1146/annurevgenet-102108-134850)

Received in final form 7 December 2018

Accepted 8 January 2019

Accepted Preprint published online 8 January 2019 https://joe.bioscientifica.com https://doi.org/10.1530/JOE-18-0673 (c) 2019 Society for Endocrinology Published by Bioscientifica Ltd. Printed in Great Britain 